\title{
AN INEQUALITY WITH APPLICATIONS TO THE SUBELLIPTICITY OF THE $\bar{\partial}$-NEUMANN PROBLEM
}

\author{
LOP-HING HO
}

(Communicated by Barbara L. Keyfitz)

\begin{abstract}
We prove an interesting inequality in this note. This inequality will be used to remove an unnecessary assumption in [2]. That paper dealt with the sufficient condition for the subellipticity of the $\bar{\partial}$-Neumann problem on nonpseudoconvex domains. We will then state the revised theorem and show why the original assumption can be removed.
\end{abstract}

Proposition 1. Let $\Omega \subset \mathbb{C}^{n}, x_{0} \in b \Omega$, and $L \in T^{1,0}(b \Omega)$. Then given any $\varepsilon>0$, there exists a neighbourhood $U$ of $x_{0}$ and $C>0$ such that for all $u$, $v \in C_{0}^{\infty}(U \cap \bar{\Omega})$ we have

$$
|(L u, v)| \leq \varepsilon\left(\|\bar{L} u\|^{2}+\|L v\|^{2}\right)+C\left(\|u\|^{2}+\|v\|^{2}\right) .
$$

Proof. We know that the adjoint $L^{*}$ of $L$ is given by

$$
L^{*}=-\bar{L}+g \text {, }
$$

where $g$ is smooth. Hence

$$
L+L^{*}=L-\bar{L}+g .
$$

By a change of coordinates we may assume that

$$
L-\bar{L}=-i a(x) \frac{\partial}{\partial x_{1}}
$$

where $a(x)>0$ in $U$. Hence the symbol of $L-\bar{L}$ is given by

$$
\sigma(L-\bar{L})=a(x) \xi_{1} .
$$

We define operators $P^{+}, P^{-}$, and $P^{0}$ as follows:

$$
\begin{aligned}
\widehat{P^{+}} u(\xi, r) & =\chi_{1}\left(\xi_{1}\right) \hat{u}(\xi, r) \\
\widehat{P^{-}} u(\xi, r) & =\chi_{2}\left(\xi_{1}\right) \hat{u}(\xi, r) \\
P^{0} & =I-P^{+}-P^{-},
\end{aligned}
$$

Received by the editors September 21, 1989.

1980 Mathematics Subject Classification (1985 Revision). Primary 35N15. 
where $\chi_{1}, \chi_{2} \in C^{\infty}(\mathbb{R}), 0 \leq \chi_{i} \leq 1, i=1,2$, and

$$
\begin{aligned}
& \chi_{1}(\xi)= \begin{cases}1 & \xi \geq 1, \\
0 & \xi<0,\end{cases} \\
& \chi_{2}(\xi)= \begin{cases}0 & \xi>0, \\
1 & \xi \leq-1 .\end{cases}
\end{aligned}
$$

We now define

$$
\{u, v\}^{+}=\left\langle\left(L+L^{*}\right) P^{+} u, P^{+} v\right\rangle+K\left\langle P^{+} u, P^{+} v\right\rangle,
$$

where $K$ is a large positive constant.

Lemma 2. $\{u, v\}^{+}$is an inner product for large $K$.

Proof. Since $a(x) \xi_{1}$ is nonnegative on the support of $\chi_{1}$, by Garding's inequality we have

$$
\left\langle(L-\bar{L}) P^{+} u, P^{+} u\right\rangle \geq-C\left\langle P^{+} u, P^{+} u\right\rangle .
$$

Hence for some large $K$ we have $\{u, u\}^{+} \geq 0$.

It is easy to see that $\{u, u\}^{+}=0$ if and only if $u=0$, and that $\{u, v\}^{+}=$ $\overline{\{v, u\}^{+}}$.

Since $\{u, v\}^{+}$is an inner product, by Schwarz's inequality

$$
\left|\{u, v\}^{+}\right| \leq\left(\{u, u\}^{+}\right)^{1 / 2}\left(\{v, v\}^{+}\right)^{1 / 2} .
$$

It is easily seen that

$$
\begin{aligned}
\{u, u\}^{+} & =\left\langle\left(L+L^{*}\right) P^{+} u, P^{+} u\right\rangle+K\left\langle P^{+} u, P^{+} u\right\rangle \\
& \leq \varepsilon\|\bar{L} u\|^{2}+C\|u\|^{2}
\end{aligned}
$$

by (1). Similarly,

$$
\{v, v\}^{+} \leq \varepsilon\|L v\|^{2}+C\|v\|^{2} .
$$

Hence

$$
\begin{aligned}
\left\langle L P^{+} u, P^{+} v\right\rangle & =\{u, v\}^{+}-\left\langle L^{*} P^{+} u, P^{+} v\right\rangle-K\left\langle P^{+} u, P^{+} v\right\rangle \\
& =\{u, v\}^{+}+\left\langle P^{+} u, L P^{+} v\right\rangle-\left\langle g P^{+} u, P^{+} v\right\rangle-K\left\langle P^{+} u, P^{+} v\right\rangle,
\end{aligned}
$$

and we get from (2), (3), and (4) that

$$
\left|\left\langle L P^{+} u, P^{+} v\right\rangle\right| \leq \varepsilon\left(\|\bar{L} u\|^{2}+\|L v\|^{2}\right)+C\left(\|u\|^{2}+\|v\|^{2}\right) .
$$

Similarly, we define

$$
\{u, v\}^{-}=-\left\langle\left(L+L^{*}\right) P^{-} u, P^{-} v\right\rangle+K\left\langle P^{-} u, P^{-} v\right\rangle .
$$

Just as above, $\{u, v\}^{-}$is an inner product, and again

$$
\left|\left\langle L P^{-} u, P^{-} v\right\rangle\right| \leq \varepsilon\left(\|\bar{L} u\|^{2}+\|L v\|^{2}\right)+C\left(\|u\|^{2}+\|v\|^{2}\right) .
$$


Finally,

(7)

$$
\begin{aligned}
\langle L u, v\rangle= & \left\langle L P^{+} u, P^{+} v\right\rangle+\left\langle L P^{-} u, P^{-} v\right\rangle+\left\langle L P^{+} u, P^{0} v\right\rangle+\left\langle L P^{+} u, P^{-} v\right\rangle \\
& +\left\langle L P^{-} u, P^{+} v\right\rangle+\left\langle L P^{-} u, P^{0}\right\rangle+\left\langle L P^{0} u, P^{+} v\right\rangle+\left\langle L P^{0} u, P^{0} v\right\rangle \\
& +\left\langle L P^{0} u, P^{-} v\right\rangle .
\end{aligned}
$$

To deal with the third to ninth terms on the right-hand side of (7), we need the facts that

$$
\|[L, P] u\|^{2} \leq \text { const }\|u\|^{2},
$$

where $P=P^{+}, P^{-}$, or $P^{0}$ and that

$$
\|(L-\bar{L}) P u\|^{2} \leq \text { const }\|u\|^{2}
$$

if the symbol of $P$ is a compactly supported function of $\xi_{1}$.

Thus, combining (5), (6), (7), and the above two facts, we get

$$
|(L u, v)| \leq \varepsilon\left(\|\bar{L} u\|^{2}+\|L v\|^{2}\right)+C\left(\|u\|^{2}+\|v\|^{2}\right) .
$$

We can now restate Theorem 2.2 in [2]. We refer the reader to [2] for the details and for definitions of $A^{(k)}$ and $I_{k}^{q}$. We assume that the reader is familiar with the $\bar{\partial}$-Neumann problem and subelliptic estimates. A detailed formulation of the problem can be found in [1] or [3].

Theorem 3. Let $\Omega$ be a domain in $\mathbb{C}^{n}$ with $C^{\infty}$ boundary, $x_{0} \in b \Omega$, and $L_{1}, \ldots, L_{n}$ a $C^{\infty}$ basis for $T^{1,0}$ so that $L_{1}, \ldots, L_{n-1}$ are tangential on $b \Omega$. Assume that there exists a neighborhood $U$ of $x_{0}$ such that for some $k \geq n-q$ the matrix $A^{(k)}$ associated with the matrix of the Levi form is positive semidefinite in $U$, then if $1 \in I_{m}^{q}\left(x_{0}\right)$ for some $m$, there is a subelliptic estimate for $(p, q)$ forms at $x_{0}$.

The following extra assumption is made in Theorem 2.2 in [2]: that

For all $\varepsilon>0$, there exists $C>0$ ( $C$ depends on $S^{0}$ but not on $\left.\phi\right)$ such

$$
\left|\left\langle D \phi_{I}, S^{0} \phi_{J}\right\rangle\right| \leq \varepsilon\left(\|\bar{\partial} \phi\|^{2}+\left\|\bar{\partial}^{*} \phi\right\|^{2}\right)+C\|\phi\|^{2}
$$

for all $\phi \in D_{U}^{p, q}$ where $D \in\left\{\overline{L_{k+1}}, \ldots, \overline{L_{n-1}}\right\}, S^{0}$ is a tangential pseudodifferential operator of order zero.

In [2] this assumption is used to verify the inequality

$$
\begin{aligned}
& \sum_{\substack{j \notin J \text { or } \\
j \in\{1, \ldots, k, n\}}}\left\|\bar{L}_{j} \phi_{J}\right\|^{2}+\sum_{\substack{j \in J \text { and } \\
k<j<n}}\left\|L_{j} \phi_{J}\right\|^{2}+\sum_{I, J} \int_{b \Omega} A_{I J}^{(k)} \phi_{I} \overline{\phi_{J}} d S \\
& \leq C\left(\|\bar{\partial} \phi\|^{2}+\left\|\bar{\partial}^{*} \phi\right\|^{2}+\|\phi\|^{2}\right)
\end{aligned}
$$


for all $\phi \in D_{U}^{p, q}$ from the inequality

$$
\begin{aligned}
& \sum_{\substack{j \notin J \text { or } \\
j \in\{1, \ldots, k, n\}}}\left\|\bar{L}_{j} \phi_{J}\right\|^{2}+\sum_{\substack{j \in J \text { and } \\
k<j<n}}\left\|L_{j} \phi_{J}\right\|^{2}+\sum_{I, J} \int_{b \Omega} A_{I J}^{(k)} \phi_{I} \bar{\phi}_{J} d S+R(\phi) \\
& \leq C\left(\|\bar{\partial} \phi\|^{2}+\left\|\bar{\partial}^{*} \phi\right\|^{2}+\|\phi\|^{2}\right),
\end{aligned}
$$

where $R(\phi)=\sum_{j=1}^{n}\left\langle\bar{L}_{j} \phi_{J}, h_{j} \phi_{K}\right\rangle+O\left(\|\phi\|^{2}\right)$ for some smooth functions $h_{j}$.

We prove the following to remove assumption (8):

Lemma 4. The inequality (9) is true without assumption (8).

Proof. We want to prove (9) using (10) and Proposition 1. Clearly what we need to prove is that for all $j=1,2, \ldots, n$,

$$
\left|\left\langle\bar{L}_{j} \phi_{J}, h_{j} \phi_{K}\right\rangle\right| \leq \varepsilon\left(\sum_{\substack{j \notin J \text { or } \\ j \in\{1, \ldots, k, n\}}}\left\|\bar{L}_{j} \phi_{J}\right\|^{2}+\sum_{\substack{j \in J \text { and } \\ k<j<n}}\left\|L_{j} \phi_{J}\right\|^{2}\right)+C\|\phi\|^{2} .
$$

When $j \in\{1,2, \ldots, k, n\}$, we have

$$
\left|\left\langle\bar{L}_{j} \phi_{J}, h_{j} \phi_{K}\right\rangle\right| \leq \varepsilon\left\|\bar{L}_{j} \phi_{J}\right\|^{2}+C\|\phi\|^{2} \text {. }
$$

When $j \in\{k+1, \ldots, n-1\}$, if $j \notin J$ or $j \in K$, then $\left\|\bar{L}_{j} \phi_{J}\right\|^{2}$ or $\left\|L_{j} \phi_{K}\right\|^{2}$ is in the right-hand side of (11). Hence, using the type of inequality in (12) or by integrating $\bar{L}_{j}$ by parts on the left-hand side, we can absorb the term $\left\langle\bar{L}_{j} \phi_{J}, h_{j} \phi_{K}\right\rangle$ in the right-hand side of (11). Finally, when $j \in J$ and $j \notin K$, we use Proposition 1 , and we have

$$
\left|\left\langle\bar{L}_{j} \phi_{J}, h_{j} \phi_{K}\right\rangle\right| \leq \varepsilon\left(\left\|\bar{L}_{j} \phi_{J}\right\|^{2}+\left\|L_{j} \phi_{J}\right\|^{2}\right)+C\|\phi\|^{2} \text {. }
$$

We see that the terms in the right-hand side of the above inequality are in the right-hand side of (11). This finishes the proof.

\section{REFERENCES}

1. G. B. Folland and J. J. Kohn, The Neumann problem for the Cauchy-Riemann complex, Ann. of Math. Stud., no. 75, Princeton University Press, Princeton, NJ, 1972.

2. L. Ho, Subellipticity of the $\bar{\partial}-$ Neumann problem on nonpseudoconvex domains, Trans. Amer. Math. Soc. 291 (1985), 43-73.

3. J. J. Kohn, Subellipticity of the $\bar{\partial}$-Neumann problem on pseudoconvex domains: sufficient conditions, Acta Math. 142 (1979), 79-122.

Department of Mathematics, The Wichita State University, Wichita, Kansas 67208 